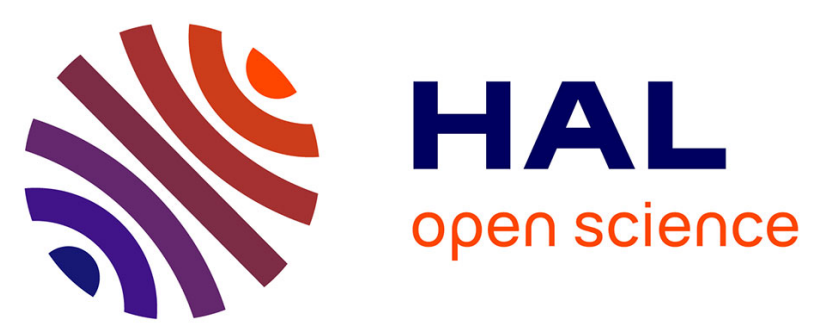

\title{
Direct observation of magnetization reversal and low field magnetoresistance of epitaxial La0.7Sr0.3MnO3/SrTiO3 (001) thin films at room temperature
}

Dalal Fadil, Sheng Wu, B. Renault, Mohammed Saib, Sylvain Lebargy, Julien Gasnier, Bruno Guillet, Jean-Marc Routoure, Stéphane Flament, Laurence Méchin

\section{- To cite this version:}

Dalal Fadil, Sheng Wu, B. Renault, Mohammed Saib, Sylvain Lebargy, et al.. Direct observation of magnetization reversal and low field magnetoresistance of epitaxial La0.7Sr0.3MnO3/SrTiO3 (001) thin films at room temperature. Journal of Applied Physics, 2012, 112, pp.013906. 10.1063/1.4730966 . hal-00976072

\section{HAL Id: hal-00976072 https://hal.science/hal-00976072}

Submitted on 9 Apr 2014

HAL is a multi-disciplinary open access archive for the deposit and dissemination of scientific research documents, whether they are published or not. The documents may come from teaching and research institutions in France or abroad, or from public or private research centers.
L'archive ouverte pluridisciplinaire HAL, est destinée au dépôt et à la diffusion de documents scientifiques de niveau recherche, publiés ou non, émanant des établissements d'enseignement et de recherche français ou étrangers, des laboratoires publics ou privés. 


\section{AIP Applied Physics}

\section{Direct observation of magnetization reversal and low field magnetoresistance of epitaxial La0.7Sr0.3MnO3/SrTiO3 (001) thin films at room temperature}

D. Fadil, S. Wu, P. Perna, B. Renault, M. Saïb et al.

Citation: J. Appl. Phys. 112, 013906 (2012); doi: 10.1063/1.4730966

View online: http://dx.doi.org/10.1063/1.4730966

View Table of Contents: http://jap.aip.org/resource/1/JAPIAU/v112/i1

Published by the American Institute of Physics.

\section{Related Articles}

Easy-plane magnetocrystalline anisotropy of compressive strained (La, Ba)MnO3 film J. Appl. Phys. 112, 013913 (2012)

Domain-related origin of magnetic relaxation in compressively strained manganite thin films Appl. Phys. Lett. 101, 012408 (2012)

High propagating velocity of spin waves and temperature dependent damping in a CoFeB thin film Appl. Phys. Lett. 100, 262412 (2012)

Direct evidence for stress-induced (001) anisotropy of rapid-annealed FePt thin films

Appl. Phys. Lett. 100, 261909 (2012)

Crystalline anisotropic magnetoresistance with two-fold and eight-fold symmetry in (In,Fe)As ferromagnetic semiconductor

Appl. Phys. Lett. 100, 262409 (2012)

\section{Additional information on J. Appl. Phys.}

Journal Homepage: http://jap.aip.org/

Journal Information: http://jap.aip.org/about/about_the_journal

Top downloads: http://jap.aip.org/features/most_downloaded

Information for Authors: http://jap.aip.org/authors

\section{ADVERTISEMENT}

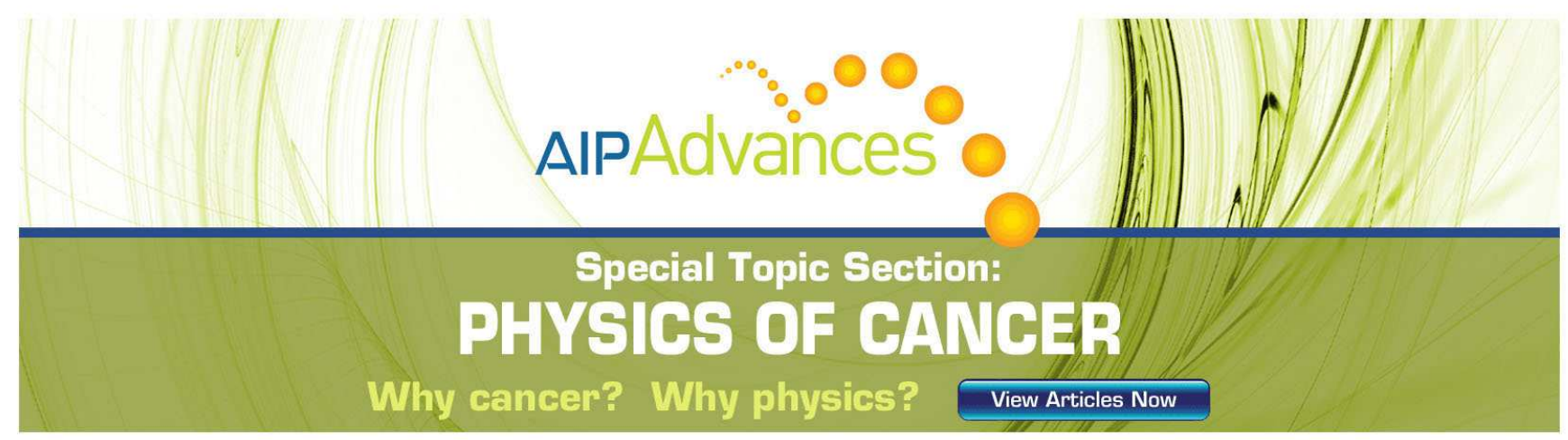




\title{
Direct observation of magnetization reversal and low field magnetoresistance of epitaxial $\mathrm{La}_{0.7} \mathrm{Sr}_{0.3} \mathrm{MnO}_{3} / \mathrm{SrTiO}_{3}(001)$ thin films at room temperature
}

\author{
D. Fadil, ${ }^{1}$ S. Wu, ${ }^{1}$ P. Perna, ${ }^{1,2}$ B. Renault, ${ }^{1}$ M. Saïb, ${ }^{1}$ S. Lebargy,${ }^{1}$ J. Gasnier, ${ }^{1}$ B. Guillet, ${ }^{1}$ \\ J.-M. Routoure, ${ }^{1}$ S. Flament, ${ }^{1}$ and L. Méchin ${ }^{1}$ \\ ${ }^{1}$ GREYC (UMR 6072), CNRS, ENSICAEN, Université de Caen Basse-Normandie, 6 Boulevard Maréchal Juin, \\ 14050 Caen Cedex, France \\ ${ }^{2}$ Instituto Madrileño de Estudios Avanzados en Nanociencia, IMDEA-Nanociencia, Campus Universidad \\ Autónoma de Madrid, 28049 Madrid, Spain
}

(Received 16 March 2012; accepted 24 May 2012; published online 5 July 2012)

\begin{abstract}
We have observed the in-plane magnetic domain arrangement during magnetization reversal in a $40 \mathrm{~nm}$ thick $\mathrm{La}_{0.7} \mathrm{Sr}_{0.3} \mathrm{MnO}_{3} / \mathrm{SrTiO}_{3}$ (001) thin film patterned into $500 \mu \mathrm{m}$ long microbridges of width 50 or $100 \mu \mathrm{m}$. Magneto-optical Kerr effect microscopy was used at room temperature and magnetic hysteresis loops were deduced from local averaging of intensity over the microbridge areas. Magnetization reversal proceeds by nucleation and propagation of $180^{\circ}$ domain walls. When the magnetic field was applied parallel to the bridge, we observed the nucleation of only one or two domain walls and the reversal occurred by the propagation of them. When the magnetic field was applied perpendicular to the bridge, the reversal occurred mostly by the nucleation of several domain walls. The low field magnetoresistance (MR) and the low frequency noise at zero magnetic field were measured at room temperature. In addition to the linear and reversible colossal MR effect, hysteretic MR versus magnetic field curves could be observed, showing two maxima (minima) when the magnetic field is parallel (perpendicular) to the bridge length. The observed hysteretic MR behaviour is attributed to anisotropic MR inside the $180^{\circ}$ Néel domain walls. (C) 2012 American Institute of Physics. [http://dx.doi.org/10.1063/1.4730966]
\end{abstract}

\section{INTRODUCTION}

Because of its large spin polarization and its high Curie temperature, $\mathrm{La}_{0.7} \mathrm{Sr}_{0.3} \mathrm{MnO}_{3}$ (LSMO) is promising for a large number of applications. ${ }^{1-3}$ The magnetoresistance (MR) is generally defined as

$$
\mathrm{MR}(\mathrm{H})=\frac{\mathrm{R}(\mathrm{H})-\mathrm{R}(0)}{\mathrm{R}(0)}
$$

where $\mathrm{H}$ is the magnetic field, $\mathrm{R}(\mathrm{H})$ is the sample electrical resistance in magnetic field, and $R(0)$ is the sample electrical resistance in zero magnetic field. In the vicinity of the Curie temperature $\mathrm{T}_{\mathrm{C}}$, LSMO exhibits the so-called colossal magnetoresistance (CMR) effect. ${ }^{4-8}$ The latter is an intrinsic phenomenon and is observed at high magnetic field (several tesla), thus limiting possible device applications. At moderately low magnetic field $(<0.1 \mathrm{~T})$, MR effects are referred to as low field magnetoresistance (LFMR). In contrast to CMR, LFMR can find applications in magnetic sensors. A recent review of low-field magnetotransport in manganites can be found in Ref. 9. Table I summarizes some literature data reporting measured values of LFMR in LSMO single layers with various structural properties: epitaxial, polycrystalline or with grain boundaries (GB). The intrinsic LFMR in epitaxial LSMO films is relatively small as compared to other ferromagnetic materials. ${ }^{10,11}$ Polycrystalline samples can be used to enhance the LFMR value, taking advantage of spinpolarized intergrain tunnelling at the GB regions. ${ }^{12}$ At room temperature, LFMR is about $-0.05 \%$ at $5 \mathrm{mT}$ in epitaxial LSMO films deposited on $\mathrm{SrTiO}_{3}$ (STO) (001) substrates, and it is increased by a factor of about 10 in LSMO films deposited on polycrystalline yttria-stabilized-zirconia (YSZ) substrates. ${ }^{10}$ It was also reported that at $4.2 \mathrm{~K}$ an increase of MR by a factor 60 in LSMO films on polycrystalline STO $(-15 \%$ at $150 \mathrm{mT})$ compared to that measured in epitaxial LSMO films on STO (001). ${ }^{11}$ However, polycrystalline materials always show additional electrical noise compared to epitaxial films and should therefore be considered with care in view of magnetic sensor applications. ${ }^{13}$ Other extrinsic MR effects are studied to enhance the LFMR values, mainly based on artificial GB that are engineered by using bicrystal substrates, ${ }^{14-16}$ step-edge structures, ${ }^{17}$ and laser patterned geometries. ${ }^{18}$ Domain wall magnetoresistance in LSMO has also been studied in nanoconstrictions since large LFMR is expected in narrow domain walls. ${ }^{19-22}$

A better understanding of the magnetic domain arrangement in LSMO films during magnetization reversal and its role in the LFMR are needed in order to improve the performance of LSMO-based magnetic devices. It is also a crucial issue for an optimized operation of spin polarized devices like spin valves or magnetic tunnel junctions using LSMO as electrodes or applications in logic devices. ${ }^{1,23-25}$ Literature data about the in-plane magnetic domain arrangement during magnetization reversal in epitaxial or polycrystalline LSMO thin films are rather scarce. Using a wide-field Kerr microscope, Gupta et al. have shown that magnetization reversal occurs by domain wall motion inside grains in 
TABLE I. Literature data reporting measured values of LFMR in LSMO single layers of various structural properties. Most of the values are estimated from graphical data in cited papers.

\begin{tabular}{|c|c|c|c|c|c|}
\hline Materials & Structural properties & Film thickness (nm) & $\mathrm{T}(\mathrm{K})$ & LFMR & References \\
\hline $\mathrm{La}_{0.7} \mathrm{Sr}_{0.3} \mathrm{MnO}_{3} / \mathrm{STO}(001)$ & Epitaxial film & $150-200$ & 300 & $-0.05 \%$ at $5 \mathrm{mT}$ & 10 \\
\hline $\mathrm{La}_{0.7} \mathrm{Sr}_{0.3} \mathrm{MnO}_{3} / \mathrm{YSZ}$-poly & Polycrystalline film & $150-200$ & 300 & $-0.5 \%$ at $5 \mathrm{mT}$ & \\
\hline $\mathrm{La}_{0.67} \mathrm{Sr}_{0.33} \mathrm{MnO}_{3} / \mathrm{STO}(001)$ & Epitaxial film & 100 & 4.2 & $-0.25 \%$ at $150 \mathrm{mT}$ & 11 \\
\hline $\mathrm{La}_{0.67} \mathrm{Sr}_{0.33} \mathrm{MnO}_{3} / \mathrm{STO}$-poly & Polycrystalline film & 100 & 4.2 & $-15 \%$ at $150 \mathrm{mT}$ & \\
\hline $\mathrm{La}_{0.7} \mathrm{Sr}_{0.3} \mathrm{MnO}_{3} / \mathrm{STO}$ bicrystal $30^{\circ}$ & Bicrystalline film & 120 & 100 & $2 \%$ at $15 \mathrm{mT}$ & 15 \\
\hline $\mathrm{La}_{0.7} \mathrm{Sr}_{0.3} \mathrm{MnO}_{3} / \mathrm{STO}(001)$ & Epitaxial film on step edge patterned substrate & 70 & 299 & $-1.5 \%$ at $50 \mathrm{mT}$ & 17 \\
\hline $\mathrm{La}_{0.7} \mathrm{Sr}_{0.3} \mathrm{MnO}_{3} / \mathrm{STO}(001)$ & Epitaxial film on laser patterned substrate & 10 & 20 & $10 \%$ at $50 \mathrm{mT}$ & 18 \\
\hline
\end{tabular}

polycrystalline LSMO films while it occurs by domain wall movement in large area in epitaxial LSMO films. ${ }^{26,27}$ Lecoeur et al. observed magnetic domain orientation and contrast suggesting a biaxial magnetic anisotropy with (110) easy axes in epitaxial LSMO films at room temperature. ${ }^{27}$ The magnetization reversal process at room temperature in patterned LSMO thin films has been previously studied by our group. ${ }^{28}$ Nucleation/propagation of magnetic domain walls and pinning in the $2 \mu \mathrm{m}$ and $5 \mu \mathrm{m}$ diameter holes were observed in the $50 \mu \mathrm{m}$ long rectangles of various widths in the $20-50 \mu \mathrm{m}$ range. We also previously showed that magnetization reversal occurs either by nucleation and domain wall propagation or by coherent rotation depending on the magnetic field orientation with respect to the terraces in vicinal LSMO films. ${ }^{29}$ In a recent paper, Kim et al. studied the magnetic domain structure of LSMO nanoislands with dimensions on the order of $160-720 \mathrm{~nm}$ by using photoemission electron microscopy and magnetic force microscopy. ${ }^{30}$ Their conclusions were that single domain states could be stabilized with (110)-oriented LSMO elongated hexagonal islands. Rhensius et al. also investigated the interplay between the governing magnetic energy terms in patterned LSMO elements. ${ }^{31}$ Using direct high-resolution x-ray magnetic microscopy they showed that the magnetic configurations evolve from multidomain to flux-closure states with decreasing element size, which demonstrates that the control of the spin state in LSMO elements is possible by judicious choice of the geometry.

The objective of the present work is to study the LFMR at room temperature in a patterned epitaxial LSMO thin film deposited on STO (001) in relation with the magnetization reversal process observed by longitudinal magneto-optical Kerr effect (MOKE) microscopy. In Sec. II, we will describe the experimental conditions: thin film deposition, patterning into $500 \mu \mathrm{m}$ long microbridges, MOKE, MR, and low frequency noise measurement set-ups. The study of the magnetization reversal process with the magnetic field applied either parallel or perpendicular to the bridge length will be presented in Sec. III. MR measurements will be described and analysed in Sec. IV. More particularly, the results will be analysed in terms of anisotropic magnetoresistance (AMR) effect, which reflects the effect of the angle between magnetization and current direction on MR. The evaluation of the expected performance of such LSMO-based magnetoresistive sensors will finally be made by considering the signal-to-noise ratio in the low frequency range in Sec. V. A conclusion will be given in Sec. VI.

\section{EXPERIMENTAL CONDITIONS}

The $40 \mathrm{~nm}$ thick LSMO thin films were deposited by pulsed laser deposition from a stoichiometric target onto commercially available STO (001) substrates (CRYSTEC $\mathrm{GmbH}$ ). During deposition, the laser energy was $220 \mathrm{~mJ}$, the target-to-substrate distance was $50 \mathrm{~mm}$, the oxygen pressure was $0.35 \mathrm{mbar}$, and the substrate temperature was $720^{\circ} \mathrm{C}$. The crystal structure was investigated by means of $\mathrm{x}$-ray diffraction. Standard $\theta-2 \theta$ scans were routinely performed in order to determine the LSMO out-of plane lattice parameter $(0.3855 \mathrm{~nm})$, revealing an in-plane tensile strain of about $1.28 \%$ if compared to the lattice parameter of the STO substrate $(0.3905 \mathrm{~nm})$. The full-width-half-maximum of the rocking curves around the LSMO (002) peak was about $0.2^{\circ}$, indicating the good crystalline quality of the films. The magnetization of the film was measured using a superconducting quantum interference device magnetometer. Its Curie temperature, determined from the inflection point of the magnetization versus temperature curve measured at $50 \mathrm{mT}$, was $325 \mathrm{~K}$, which is close to the bulk value. Room temperature high resolution vectorial Kerr magnetometry measurements showed that the unpatterned LSMO/STO (001) film presents a very weak uniaxial magnetic anisotropy. ${ }^{32}$

After LSMO deposition, a $200 \mathrm{~nm}$ thick gold layer was sputtered on the films in order to make low resistive fourprobe connections. The gold layer was first patterned by using a potassium iodide (KI) solution. The LSMO thin film was patterned by UV photolithography and argon ion etching to form the patterned geometries. LSMO is therefore present under the gold electrodes. We fabricated microbridges of two different widths $\mathrm{W}(50 \mu \mathrm{m}$ and $100 \mu \mathrm{m})$ with the length along the (110) direction (Figure 1). The red dotted lines define the surfaces used for the calculation of normalized magnetization reported in Figure 4. Magnetoresistance and low-frequency noise measurements were performed at room temperature in a four probe configuration. The current and voltage pads $\left(\mathrm{I}^{+}, \mathrm{I}^{-}, \mathrm{V}^{+}, \mathrm{V}^{-}\right)$are shown in Figures $1(\mathrm{a})$ and 1(b). The distance between current and voltage electrodes are $500 \mu \mathrm{m}$ and $300 \mu \mathrm{m}$, respectively.

In order to observe the in-plane magnetic domain arrangement we used a home-made MOKE microscope in 


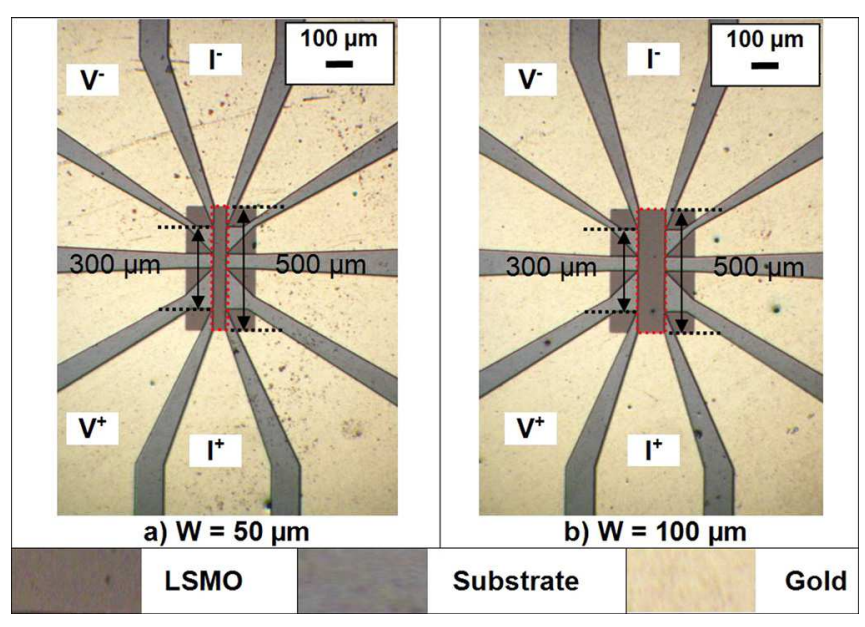

FIG. 1. Optical microscope photography of (a) the $50 \mu \mathrm{m}$ and (b) the $100 \mu$ m wide microbridges. Symbols $\left(\mathrm{I}^{-}, \mathrm{I}^{+}\right)$and $\left(\mathrm{V}^{-}, \mathrm{V}^{+}\right)$show the current and voltage gold pads used in this study, respectively. The red dotted lines define the surfaces used for the calculation of normalized magnetization reported in Figure 4. The distance between current and voltage electrodes are $500 \mu \mathrm{m}$ and $300 \mu \mathrm{m}$, respectively.

the longitudinal configuration, in which the sample holder is inclined by $45^{\circ}$ with respect to both the incident light and the detector. ${ }^{28}$ The sample was placed between the poles of an electromagnet. The magnetic field was applied in the plane of the sample. The region in depth that contributes to the Kerr rotation is at least $60 \mathrm{~nm}$ in LSMO. ${ }^{33}$ The light source was a light emitting diode at $455 \mathrm{~nm}$ (Luxeon ${ }^{\mathrm{TM}} 1$-Watt Emitter). A polarizer was inserted in the light beam to set the linear polarization of the incident light either in or perpendicular to the incidence plane, which corresponds to a p-wave or a s-wave, respectively. The detection of either the p-polarization component or the s-polarization component is achieved by appropriately tuning the configuration of the analyzer with respect to the polarizer. The area of interest was observed by using a magnifying microscope equipped with a CCD camera. The latter is a Hamamatsu 4880-80 CCD camera that operates in a 14-bits mode and produces images with $656 \times 494$ pixels over a sensitive area of $6.52 \mathrm{~mm} \times 4.89 \mathrm{~mm}^{2}$. The intensity of each pixel, which is related to the magnetization of the samples, is coded in grey levels. For each magnetic field value, the presented magnetic domain images were obtained by dividing the measured image to the same reference image acquired in the saturated state. The local hysteretic loops were deduced from MOKE images at each applied field by averaging the intensity over the $\mathrm{W} \times 500 \mu \mathrm{m}$ LSMO surface (see Figure 1). Then, the normalized intensity values, which are proportional to the sample magnetization, were plotted as a function of the applied magnetic field.

Low frequency noise measurements were carried out using a four probe station (Suss Microtech PM5) at room temperature while a micro-manipulated four probe station (Lakeshore EMPX-HF) equipped with an electromagnet was used for the MR measurements. In the latter case, the samples were placed in vacuum and the temperature was fixed at $306 \mathrm{~K} \pm 10 \mathrm{mK}$ in order to reduce electrical resistance variation due to the temperature fluctuations. The in-plane magnetic induction values $\left(\mu_{0} \mathrm{H}\right)$, where $\mu_{0}$ is the vacuum permeability, were varied in the $\pm 4 \mathrm{mT}$ range, the magnetic field step and the time between each resistance measurement was $40 \mu \mathrm{T}$ and $400 \mathrm{~ms}$, respectively. For both low frequency noise and MR measurements, the electronics readout was the same. It mainly consists of one low noise high output impedance dc current source and a dedicated low noise instrumentation amplifier with the following characteristics: A dc output dedicated to resistance measurement with a voltage gain equal to 10 and an ac output dedicated to noise measurements with a voltage gain around 1000 and a $1 \mathrm{~Hz}-1 \mathrm{MHz}$ bandwidth. ${ }^{34,35}$ The tested sample was connected at the output of the dc current source using $\mathrm{I}^{-}$and $\mathrm{I}^{+}$pads (defined in Figure 1). The sample dc voltage and the sample voltage noise were measured using $\mathrm{V}^{-}$and $\mathrm{V}^{+}$pads, thus enabling four probe measurements.

\section{MAGNETIZATION REVERSAL}

Figures 2 and 3 show the series of MOKE images recorded at room temperature during magnetization reversal in $50 \mu \mathrm{m}$ and $100 \mu \mathrm{m}$ wide patterned LSMO bridges with the magnetic field applied in the plane either parallel (Figure 2) or perpendicular (Figure 3) to the bridge length. In each case, we show the reversal from one saturated state (grey colour) to the opposite one (black or white depending if the magnetic field was increased or decreased). In all cases, magnetization reversal proceeds by nucleation and propagation of domain walls, as previously observed by vectorial Kerr magnetometry measurements. ${ }^{32}$ When the magnetic field is applied parallel to the bridge length, we could observe that propagation is dominant (Figure 2), whereas when the magnetic field is applied perpendicular to the bridge length, nucleation is dominant (Figure 3).

In the $50 \mu \mathrm{m}$ wide bridge with $\mathrm{H}$ applied parallel to the bridge length (Figure 2(a)), one $180^{\circ}$ head-to-head domain wall is nucleated at one side and propagates towards the other side. We notice that between $0.470 \mathrm{mT}$ and $0.488 \mathrm{mT}$ this domain wall is pinned in the centre of the bridge, while another domain wall nucleates at the other bridge side. Independently, the domain wall nucleates and propagates in the bridge at $0.470 \mathrm{mT}$. We can also note that reversal in LSMO actually starts at $0.440 \mathrm{mT}$ outside the bridge (in the LSMO voltage pads). The nucleation of the domain walls that propagate along the bridge may occur in the current electrodes under the gold layer (not shown in the image). Saturation is reached at $0.520 \mathrm{mT}$ after the annihilation of the two domain walls. Interestingly, the observation is the same when the magnetic field is swept from positive to negative values, thus demonstrating the reproducibility of the observed magnetization reversal mechanism. In the $100 \mu \mathrm{m}$ wide bridge with $\mathrm{H}$ applied parallel to the bridge length (Figure 2(b)), two $180^{\circ}$ head-to-head domain walls are formed and one domain is nucleated at the centre of the bridge at $0.390 \mathrm{mT}$. The magnetization reversal proceeds by the propagation of the two domain walls towards the two sides of the bridge (up and down in the images). Once again, the same magnetization reversal mechanism was observed when the magnetic field was swept from positive to negative values. 


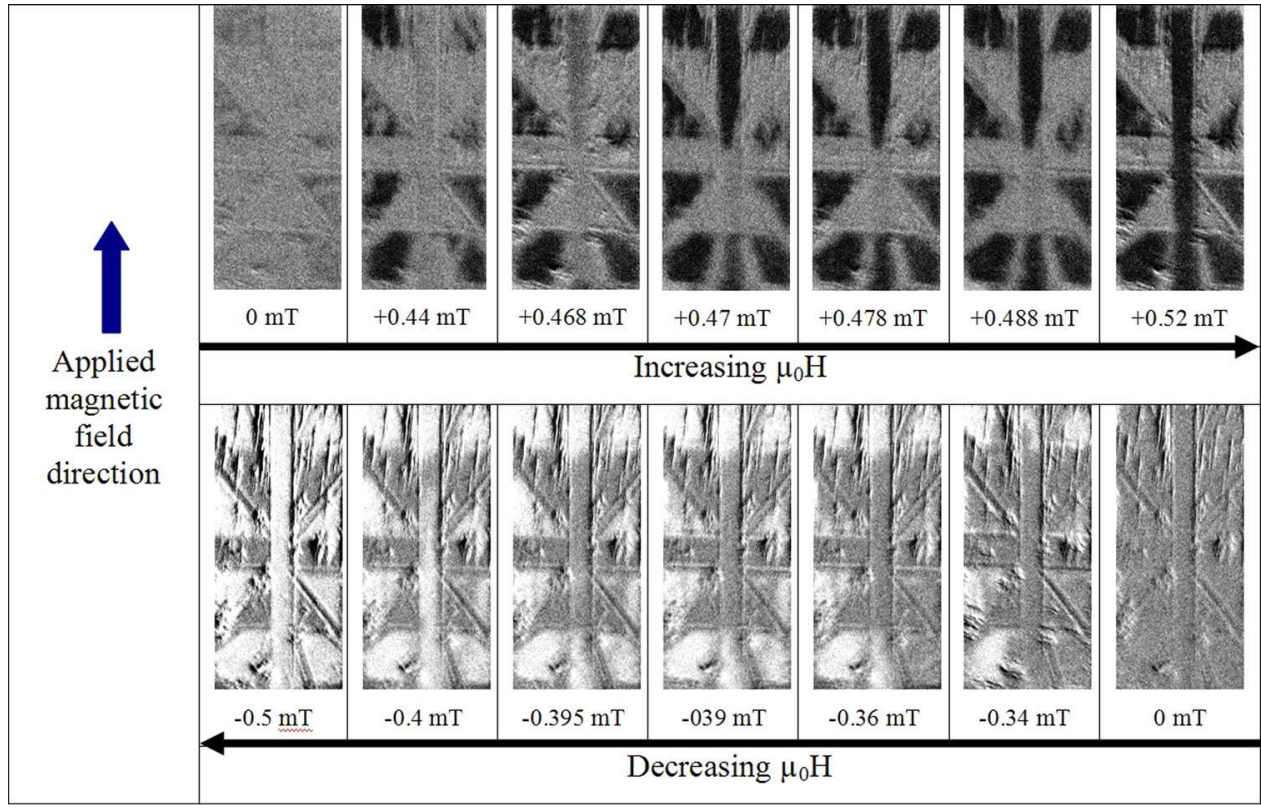

(a)

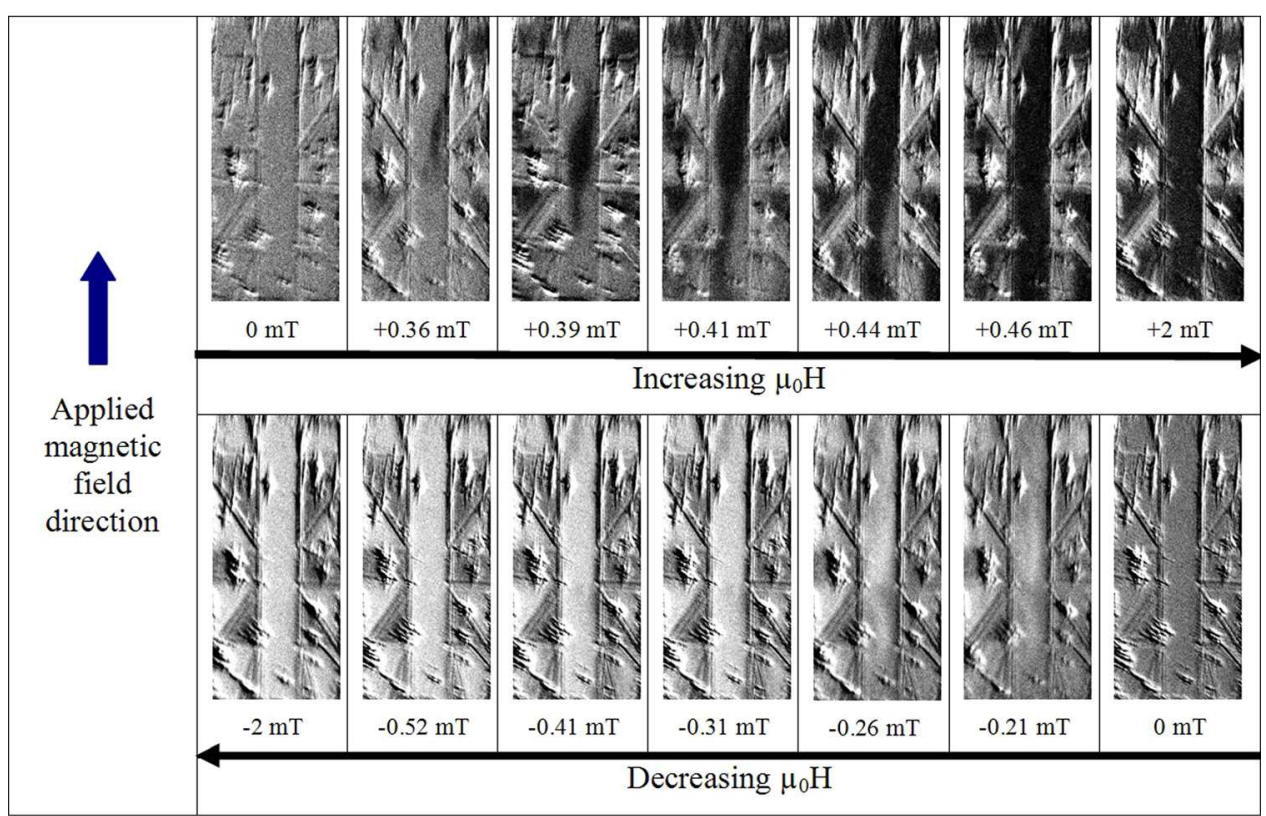

FIG. 2. Longitudinal MOKE microscopy images at room temperature during magnetization reversal in (a) the $50 \mu \mathrm{m}$ wide bridge and (b) the $100 \mu \mathrm{m}$ wide LSMO bridge when the in-plane magnetic field is applied parallel to the bridge length. The magnetic field values are indicated for each image.

(b)

In the MOKE images of Figure 3, with the applied magnetic field perpendicular to the bridge length, we can observe the presence of more magnetic domains than in Figure 2. The number of nucleation sites may be larger along the bridge length. In this case, $180^{\circ}$ domains are oriented antiparallel instead of head-to-head when the field was applied parallel to the bridge length. In the $50 \mu \mathrm{m}$ wide bridge with $\mathrm{H}$ applied perpendicular to the bridge length (Figure 3(a)), two domains nucleate at $0.208 \mathrm{mT}$. Three other domains nucleate at $0.218 \mathrm{mT}$ and even more appear at $+0.239 \mathrm{mT}$. The domain walls finally propagate to reach the saturation at $0.520 \mathrm{mT}$. In the $100 \mu \mathrm{m}$ wide bridge with $\mathrm{H}$ applied perpendicular to the bridge length (Figure 3(b)), numerous domains nucleate but the domain walls are less well defined than they were in the case of the $50 \mu \mathrm{m}$ wide bridge. The magnetization reversal occurs mainly by nucleation of many domain walls along the bridge length. When the magnetic field was swept from positive to negative values, the same observation was made in both the 50 and the $100 \mu \mathrm{m}$ wide microbridges.

Figure 4 presents the magnetic hysteresis loops versus applied magnetic field where the reported normalized magnetization is the intensity averaged over a rectangle of surface $\mathrm{W} \times 500 \mu \mathrm{m}$ (the full LSMO bridge area) in the images presented in Figures 2 and 3. When the magnetic field was applied parallel to the bridge length (dominance of domain wall propagation), the magnetic hysteresis loops are square with coercive field larger than in the case of magnetic field applied perpendicular to the bridge length (dominance of domain wall nucleation). The loop widths $2 \times \mathrm{H}_{\mathrm{c}}\left(\mathrm{H}_{\mathrm{c}}\right.$ being the coercive field) are equal to $0.860 \mathrm{mT}$ and $0.620 \mathrm{mT}$, for the $50 \mu \mathrm{m}$ and $100 \mu \mathrm{m}$ wide microbridges, respectively, when $\mathrm{H}$ is parallel to the bridge length, and $0.450 \mathrm{mT}$ and $0.400 \mathrm{mT}$, 


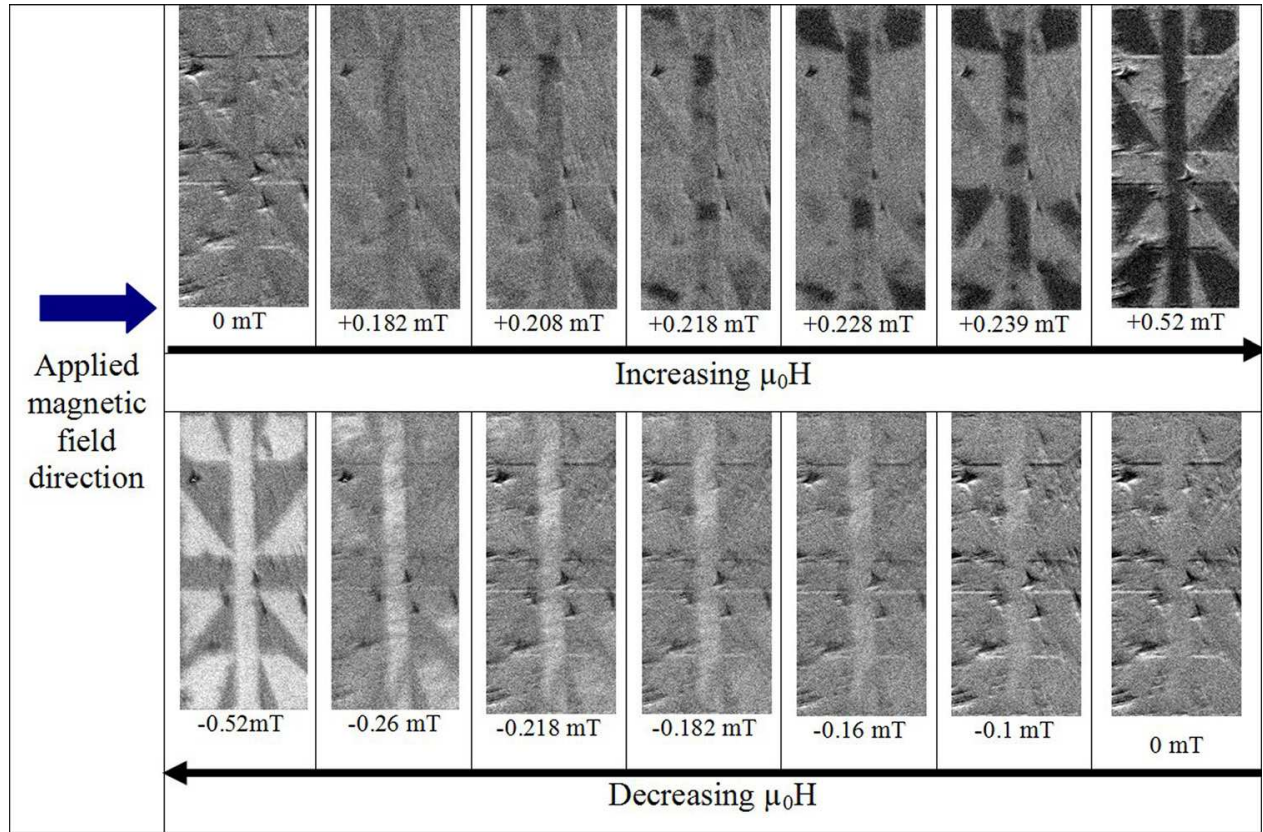

(a)

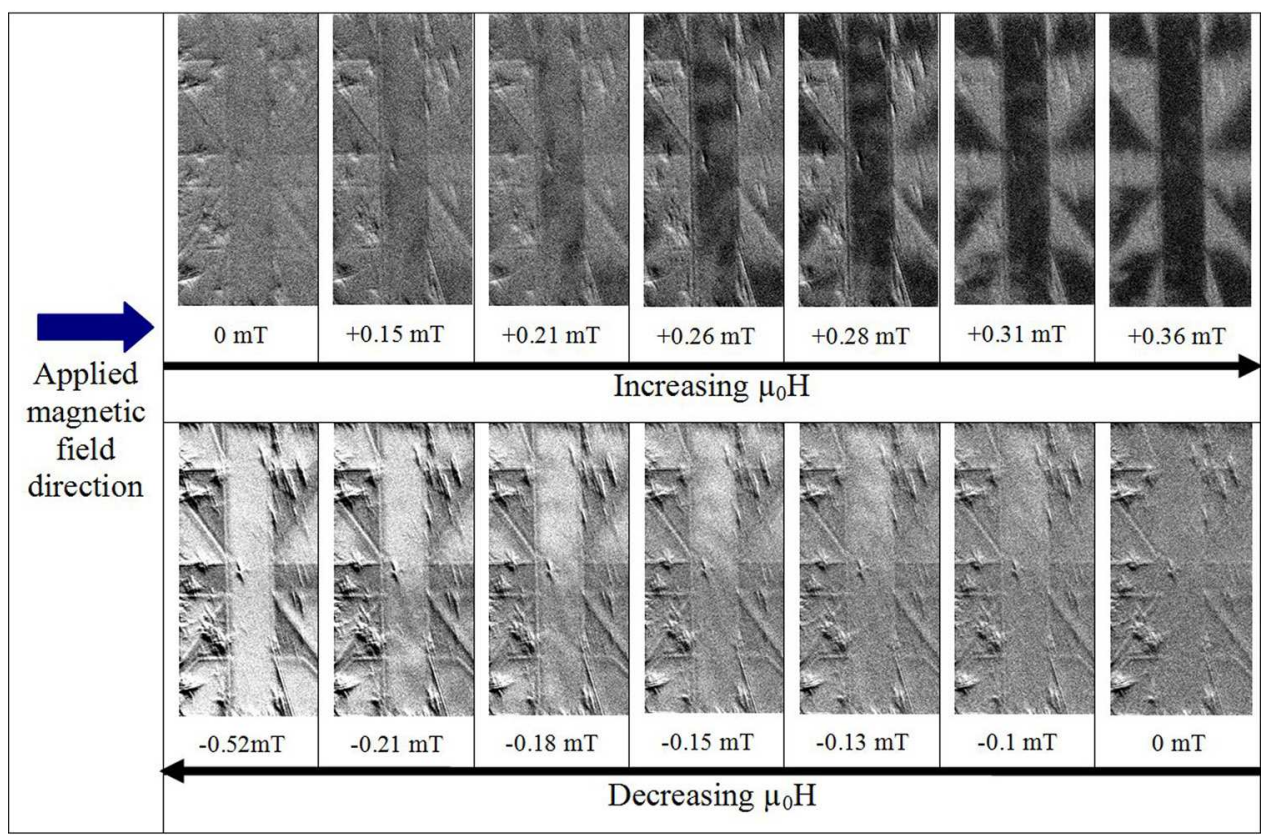

FIG. 3. Longitudinal MOKE microscopy images at room temperature during magnetization reversal in (a) the $50 \mu \mathrm{m}$ wide bridge and (b) the $100 \mu \mathrm{m}$ wide LSMO bridge when the in-plane magnetic field is applied perpendicular to the bridge length. The magnetic field values are indicated for each image.

(b)

for the $50 \mu \mathrm{m}$ and the $100 \mu \mathrm{m}$ wide microbridges, respectively, when $\mathrm{H}$ is perpendicular to the bridge length. Shape anisotropy induced by the patterned geometries in the LSMO film thus affects magnetization reversal and coercive field values in patterned LSMO as already observed by other authors. ${ }^{36}$ In addition, one would notice that the shape of the hysteresis loops is not a perfect square when $\mathrm{H}$ is perpendicular to the bridge length. By looking again carefully the MOKE images of Figure 3, one could see that some regions with different grey level appear between $0.182 \mathrm{mT}$ and $0.218 \mathrm{mT}$ (Figure 3(a)) and between $0.150 \mathrm{mT}$ and $0.210 \mathrm{mT}$ (Figure 3(b)). These grey levels may indicate that in some areas magnetization is rotating before domain walls nucleate. The contrast and magnification are not large enough to make it clear in the MOKE images but it is visible in the hysteresis loops where a linear increase of magnetization is seen as the reversal starts (Figure 4).

Figure 5 shows the time dependence of the magnetization during reversal in the $100 \mu \mathrm{m}$ wide LSMO bridge when $\mathrm{H}$ is applied parallel (Fig. 5(a)) or perpendicular (Fig. 5(b)) to the bridge length. The different curves were obtained by recording the MOKE images every $22 \mathrm{~s}$, after the sample has been placed at a fixed magnetic field (chosen in the transition zone) and by averaging the intensity over the same $100 \mu \mathrm{m}$ $\times 500 \mu \mathrm{m}$ rectangle as used for the hysteresis loops. In Figure 5(a), the curves could be fitted using an exponential equation (with about $60 \mathrm{~s}$ time constant), which is consistent with propagation driven magnetization reversal mechanism. ${ }^{37,38}$ In 


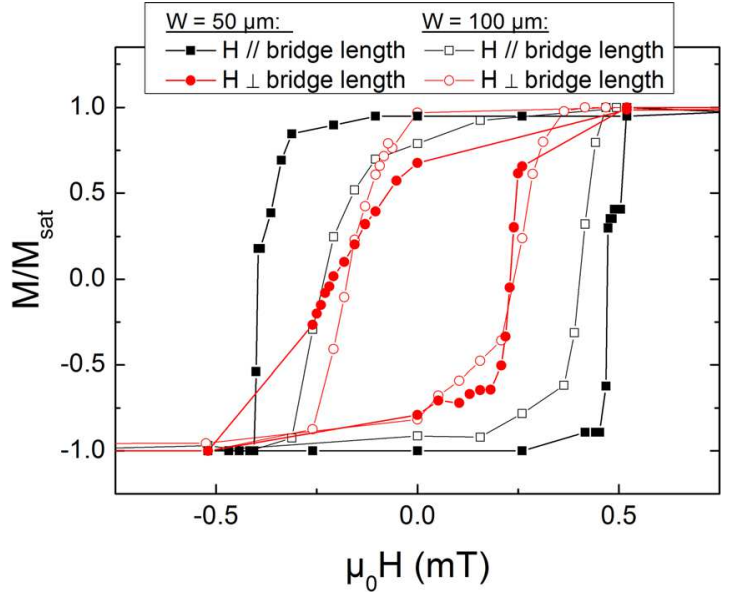

FIG. 4. Magnetic hysteresis loops versus applied magnetic field where the reported normalized magnetization is the intensity averaged over a rectangle of surface $\mathrm{W} \times 500 \mu \mathrm{m}$ (the full LSMO bridge area) in the images presented in Figures 2 and 3 for the two bridge widths $\mathrm{W}=50 \mu \mathrm{m}$ and $\mathrm{W}=100 \mu \mathrm{m}$.

Figure 5(b), no exponential fit could be proposed as expected when a nucleation driven magnetization reversal mechanism occurs.

\section{MAGNETORESISTANCE VERSUS MAGNETIC FIELD}

Figures 6(a) and 6(b) show the MR versus magnetic field characteristics at room temperature measured in the $50 \mu \mathrm{m}$ and $100 \mu \mathrm{m}$ wide LSMO bridges when the field is applied parallel or perpendicular to the bridge length. The MR shows a linear and reversible behaviour when $\left|\mu_{0} \mathrm{H}\right|>1 \mathrm{mT}$ (i.e., when the LSMO film magnetization has reached the saturation states) associated with the CMR effect. However, the MR shows a hysteretic behaviour at low field during magnetization reversal. In both the $50 \mu \mathrm{m}$ and the $100 \mu \mathrm{m}$ wide LSMO bridges, two maxima are observed when the magnetic field is parallel to the bridge length (i.e., to the bias current) and two minima are observed when the magnetic field is perpendicular to the bridge length (i.e., to the bias current). We attribute this behaviour to AMR, which reflects the dependence of the electrical resistivity on the angle between magnetization and current direction. The observed effect is opposite to those observed in more conventional ferromagnetic films, such as $\mathrm{Co}$ and $\mathrm{Ni}$, for example, where minima (maxima) were observed when $\mathrm{H}$ was applied parallel (perpendicular) to the current direction. ${ }^{39}$ AMR in ferromagnetic $3 \mathrm{~d}$ alloys is well described by theoretical models. ${ }^{40}$ In these materials, AMR is attributed to spin orbit coupling and d band splitting, which cannot be applicable to manganites, since their band structures are different. ${ }^{41}$ Other authors also reported a larger electrical resistivity in $\mathrm{La}_{0.7} \mathrm{Ca}_{0.3} \mathrm{MnO}_{3}$ when the magnetization is perpendicular to the electrical current $\mathrm{I}\left(\rho_{\perp}\right)$ than when the magnetization is parallel to the bias current I $\left(\rho_{/ /}\right)$, i.e., $\rho_{\perp}>\rho_{/ / .}{ }^{42,43}$ At low magnetic field, it was shown that AMR in $\mathrm{La}_{1-\mathrm{x}} \mathrm{Ca}_{\mathrm{x}} \mathrm{MnO}_{3}$ is sensitive to the strain state and that intrinsic magnetocrystalline anisotropy might play a role. ${ }^{44}$ Even if at low temperatures a simple tight-binding model
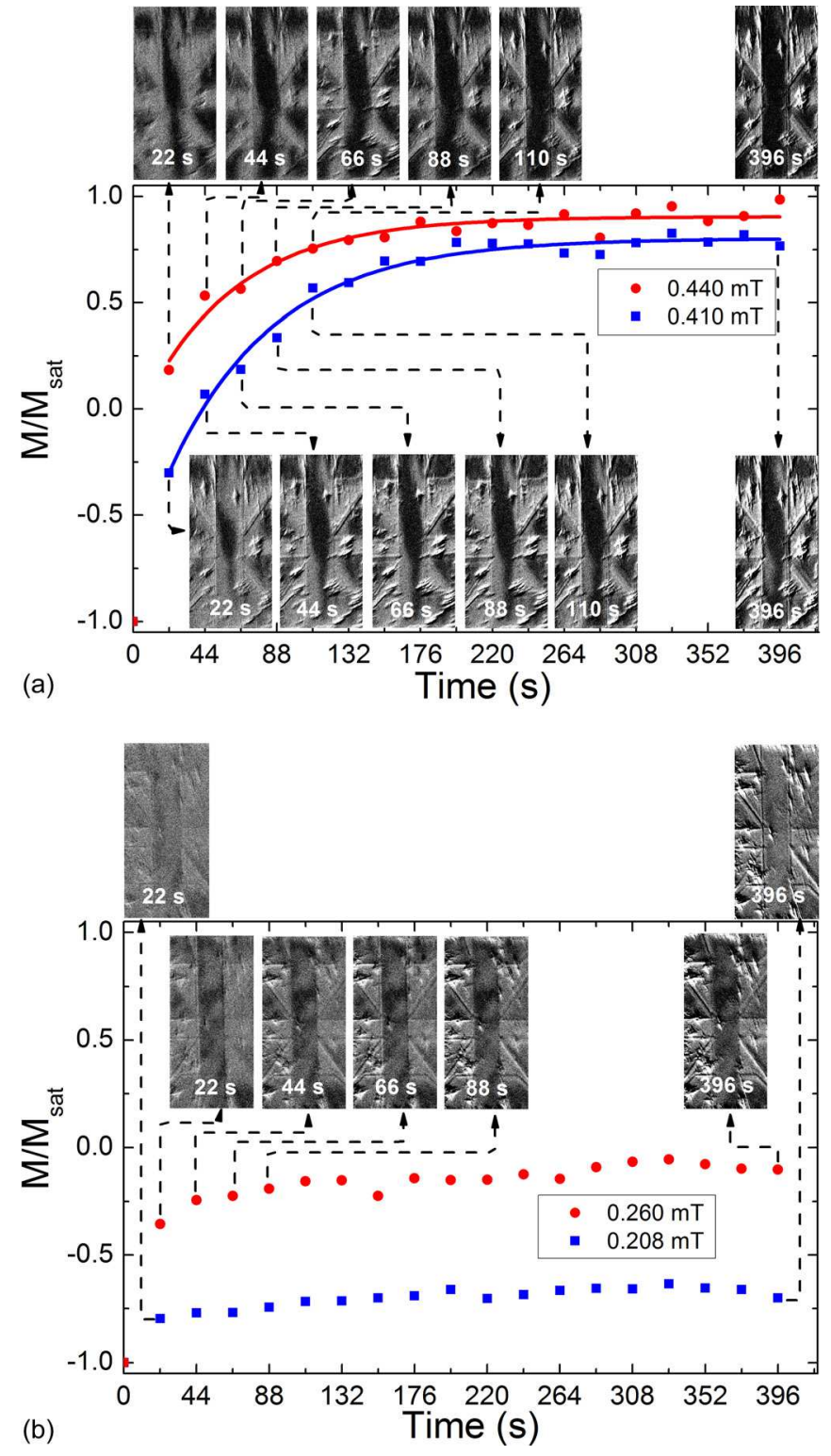

FIG. 5. Time dependence of the normalized magnetization during reversal in the $100 \mu$ m wide LSMO bridge when (a) $\mathrm{H}$ is applied parallel or (b) perpendicular to the bridge length. The different curves were obtained by recording the MOKE images every $22 \mathrm{~s}$, after the sample has been saturated and placed at a fixed magnetic field (indicated in the legends) and by averaging the intensity over the same $100 \mu \mathrm{m} \times 500 \mu \mathrm{m}$ rectangle as used for the hysteresis loops. Corresponding MOKE images are shown.

was proposed, ${ }^{45}$ an appropriate theory is still not developed to fully describe AMR in manganites, and in particular at room temperature.

Using MOKE imaging, we clearly showed that magnetization reversal in our LSMO films occurs by nucleation and propagation of domain walls, thus resulting in magnetic hysteresis loops. We then know that a multidomain configuration exists in our sample during magnetization reversal (where domains are separated by $180^{\circ}$ domain walls). A domain wall forms a continuous transition between two magnetic domains across a finite distance. The classical definition of the domain wall thickness $\delta$ depends on the material anisotropy (if the second anisotropy term is neglected, the anisotropy constant can be considered only from the first 

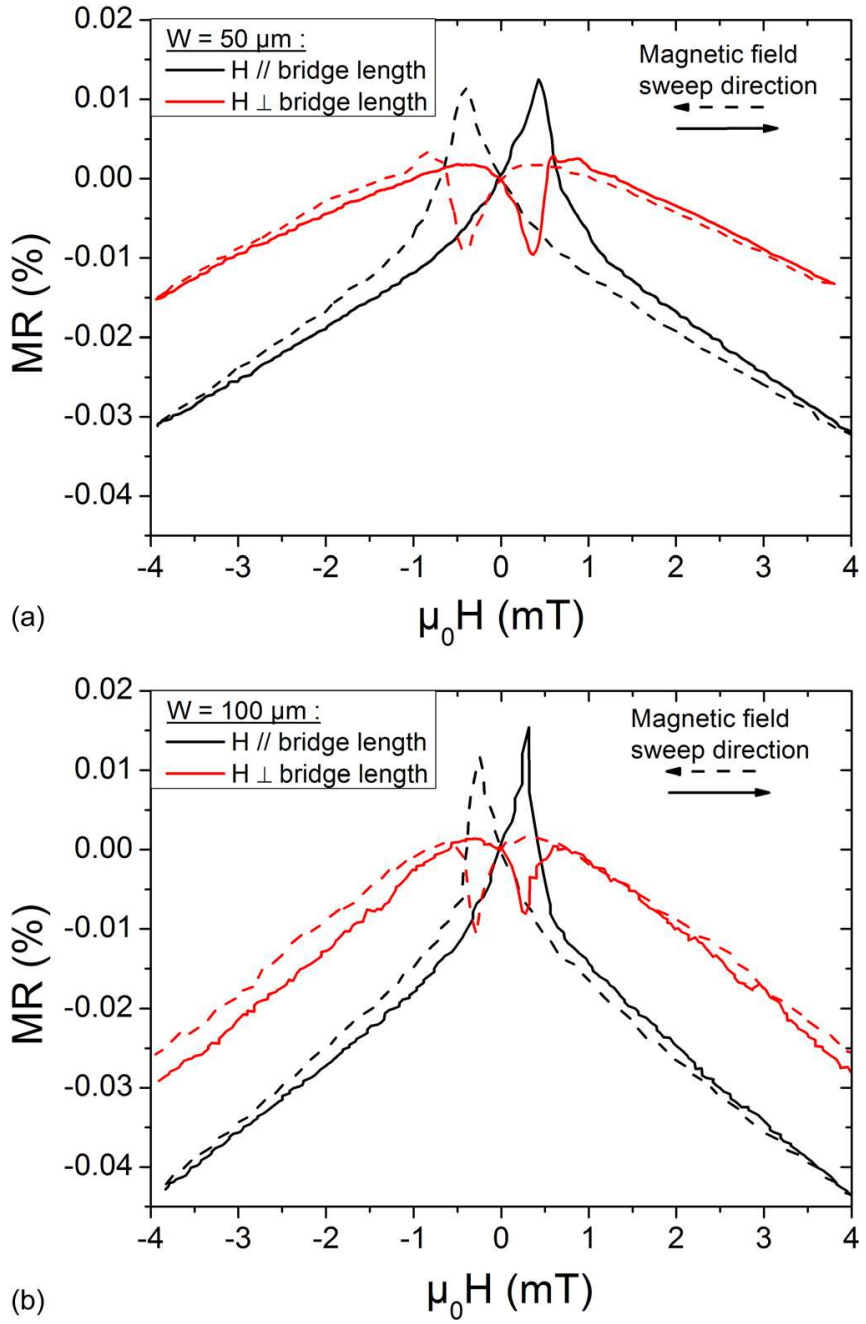

FIG. 6. Magnetoresistance versus magnetic field characteristics at room temperature measured in (a) the $50 \mu \mathrm{m}$ and (b) the $100 \mu \mathrm{m}$ wide LSMO bridges when the field is applied parallel or perpendicular to the bridge length.

term and is denoted $\mathrm{K}$ ) and the exchange constant $\mathrm{A}$ of the material, as $\delta=\pi \sqrt{\frac{\mathrm{A}}{\mathrm{K}}} \cdot 46$ For LSMO at room temperature, published values are $1.73 \times 10^{-12} \mathrm{~J} \mathrm{~m}^{-1}$ for the exchange constant A (Ref. 47) and $350 \mathrm{~J} \mathrm{~m}^{-3}$ for the anisotropy constant $\mathrm{K},{ }^{48}$ which gives a domain wall width of about $220 \mathrm{~nm}$. Magnetization inside the domain walls rotates either in the film plane (i.e., Néel walls) or out of the plane of the film (i.e., Bloch wall). From the calculation of the demagnetizing factors of the domain walls compared to the film thickness, the domain walls in our sample most probably are of Néel type. $^{46}$

The extrema in MR observed in Figure 6 can be explained in the following way. When $\mathrm{H}$ is parallel to the electrical current, magnetization is parallel (or anti-parallel) to the current direction in the saturated regions (low electrical resistivity $\left.\rho_{/ /}\right)$. During magnetization reversal, inside the head-to-head magnetic domains, magnetization is also parallel (or anti-parallel) to the current direction (low electrical resistivity $\rho_{/ /}$). Inside the $180^{\circ}$ Néel domain walls, magnetization rotates in the film plane and the average magnetization component along the direction perpendicular to the current is not zero, giving rise to high resistive regions, and therefore two maxima in the hysteretic MR loops at $\mathrm{H}_{\mathrm{c}}$ values. Comparably, when $\mathrm{H}$ is perpendicular to the bias current, magnetization is perpendicular (or anti-perpendicular) to the current direction in the saturated regions (high electrical resistivity $\rho_{\perp}$ ). During magnetization reversal, inside the anti-parallel magnetic domains, magnetization is also perpendicular (or anti-perpendicular) to the current direction (high electrical resistivity $\rho_{\perp}$ ) but inside domain walls, the average magnetization component along the direction parallel to the current is not zero, giving rise to low resistive regions, and therefore two minima in the hysteretic MR loops at $\mathrm{H}_{\mathrm{c}}$ values.

O'Donnell et al. ${ }^{42}$ also reported similar $\operatorname{MR}\left(\mu_{0} \mathrm{H}\right)$ hysteretic curves in $\mathrm{La}_{0.7} \mathrm{Ca}_{0.3} \mathrm{MnO}_{3}$ thin films showing maxima (minima) when $\mathrm{H}$ was applied parallel (perpendicular) to the current in a limited temperature range in the vicinity of $\mathrm{T}_{\mathrm{C}}$. In the analysis, they excluded a single-domain configuration and proposed a multidomain configuration with the nucleation and growth of transverse domains. They proposed that the observed hysteretic behaviour may originate from AMR in the transverse domains. Their model could also be valid if Néel type domain walls were considered and is therefore consistent with our observations.

\section{MAGNETIC SENSITIVITY AND LOW FREQUENCY NOISE MEASUREMENTS}

In addition to the reported MR values, we also calculated the magnetic sensitivity $\mathrm{S}$, which is an interesting parameter in view of magnetic sensor applications. We defined the sensitivity $\mathrm{S}$ (expressed in $\mathrm{T}^{-1}$ ) as the slope of the magnetoresistance versus magnetic field:

$$
\mathrm{S}=\left(\frac{\partial \mathbf{M R}}{\partial \mu_{0} \mathrm{H}}\right)
$$

We can determine the maximum values of $S$, named $S_{\text {max }}$, in a defined magnetic field range for all the studied configurations. No large variation of $S_{\max }$ can be found in the two investigated microbridges for the two magnetic field orientations. $S_{\max }$ values of about $\pm 1 \mathrm{~T}^{-1}$ were found at $\mu_{0} \mathrm{H}$ values of about $\pm 0.4 \mathrm{mT}$ in the $100 \mu \mathrm{m}$ wide bridge and $\pm 0.5 \mathrm{mT}$ in the $50 \mu \mathrm{m}$ wide bridge. At the same magnetic field value, the sign of $S_{\max }$ is found to be opposite depending on the magnetic field orientation with respect to the bridge length. This latter property is very interesting because it allows building a full Wheatstone bridge in a push-pull configuration leading to better performance of the sensor, such as increase of the sensitivity, rejection of the power supply perturbations and thermoresistive effects, better linearity of the output signal, etc.

Noise measurements at room temperature have been performed in demagnetized samples. The noise spectra typically consist of two parts: a low frequency noise that depends on the bias current and the frequency, and the white part that is bias current and frequency independent. The voltage noise spectral density of the latter component is equal to $4 \mathrm{k}_{\mathrm{B}} \mathrm{TR}$, with $\mathrm{k}_{\mathrm{B}}$ the Boltzmann constant, $\mathrm{T}$ the temperature, and $\mathrm{R}$ the sample electrical resistance. We used the semi-empirical 
Hooge relation to describe the low frequency noise in our sample: ${ }^{49}$

$$
\frac{\mathrm{e}_{\mathrm{nR}}^{2}}{\mathrm{~V}^{2}}=\frac{\alpha_{\mathrm{H}}}{\mathrm{n}} \times \frac{1}{\Omega \times \mathrm{f}^{\gamma}},
$$

where $\mathrm{e}_{\mathrm{nR}}^{2}$ is the voltage noise spectral density $\left(\mathrm{V}^{2} \mathrm{~Hz}^{-1}\right)$, $\mathrm{V}$ is the sample voltage $(\mathrm{V}), \alpha_{\mathrm{H}}$ is the Hooge parameter (dimensionless), $\mathrm{n}$ is the charge carrier concentration $\left(\mathrm{m}^{-3}\right)$, $\Omega$ is the sample volume $\left(\mathrm{m}^{3}\right), \mathrm{f}$ is the measuring frequency $(\mathrm{Hz})$, and $\gamma$ is the frequency slope. $\alpha_{\mathrm{H}} / \mathrm{n}$ is defined as the normalized Hooge parameter and is expressed in $\left(\mathrm{m}^{3}\right)$. The parameter $\gamma$ was checked to be equal to 1 so that we can name "1/f noise" the low frequency noise. After the quadratic dependence of $\mathrm{e}_{\mathrm{nR}}^{2}$ with the sample voltage was checked, we could estimate the normalized Hooge parameter $\alpha_{\mathrm{H}} / \mathrm{n}$ to be about $1 \times 10^{-30} \mathrm{~m}^{3}$ in our $40 \mathrm{~nm}$ thick LSMO film, which is typical of epitaxial LSMO films on STO (001) substrates. ${ }^{34}$ For example, if we consider $S_{\max }=1 \mathrm{~T}^{-1}$ at about $0.3 \mathrm{mT}$ as the average absolute value of the maximum sensitivity in the $100 \mu \mathrm{m}$ wide LSMO microbridge (of electrical resistance at zero field $\mathrm{R}(0)=2.9 \mathrm{k} \Omega$ and at a maximum current applied so that the sample voltage is $5 \mathrm{~V}$, the equivalent magnetic noise spectral density level $b_{n}$ can be estimated as the ratio of the voltage noise spectral density $\mathrm{e}_{\mathrm{n}}$ in $\mathrm{V} \mathrm{Hz}^{-1 / 2}$ over the sensitivity in $\mathrm{V} \mathrm{T}^{-1}$ within the sensor bandwidth as

$$
b_{n}=\frac{e_{n}}{S_{\max } \times V}=\frac{\sqrt{4 k_{B} T R+\frac{\left(\alpha_{H} / n\right) \times V^{2}}{\Omega \times f}}}{S_{\max } \times V} .
$$

At $\mathrm{I}_{\max }=5 \mathrm{~V} / 2.9 \mathrm{k} \Omega$, and $\mu_{0} \mathrm{H}=0.3 \mathrm{mT}, \mathrm{b}_{\mathrm{n}}$ is $29 \mathrm{nT}$ $\mathrm{Hz}^{-1 / 2}$ at $1 \mathrm{~Hz}$ and $1.4 \mathrm{nT} \mathrm{Hz}^{-1 / 2}$ in the white noise frequency range, which is close to the performance of Hall sensors $^{50}$ or commercial GMR sensors ${ }^{51}$ but still higher than the best room temperature magnetic sensors, such as long type magnetostrictive-piezoelectric laminated composite sensors (10 p Hz $\mathrm{H}^{-1 / 2}$ at $1 \mathrm{~Hz}$ (Ref. 52)), or fluxgate sensors (29 pT $\mathrm{Hz}^{-1 / 2}$ at $1 \mathrm{~Hz}$ (Ref. 53)), or giant magneto-impedance sensors (17 pT Hz ${ }^{-1 / 2}$ (Ref. 54)). LSMO thin film based magnetoresistive devices can be seen as an alternative to giant magnetoresistance and tunnel magnetoresistance, since these magnetic sensors are known to show high low frequency noise. ${ }^{55}$ The reported $b_{n}$ values in our LSMO microbridges are promising since there is still room for improvement, not only in the sensor design, such as width, length, and film thickness optimization but also in the electronic readout by the use of Wheatstone bridges, for example.

\section{CONCLUSION}

We have studied the in-plane magnetic domain structure during magnetization reversal at room temperature in patterned $40 \mathrm{~nm}$ thick LSMO/STO (001) thin films using MOKE microscopy. In all cases, magnetization reversal proceeds by nucleation and propagation of domain walls. Depending on the direction of the applied magnetic field with respect to the microbridge length, we could obtain either a dominance of domain wall propagation (when $\mathrm{H}$ is parallel to the bridge length) or a nucleation driven magnetization reversal mechanism (when $\mathrm{H}$ is perpendicular to the bridge length). In addition to the linear and reversible CMR effect, hysteretic MR curves versus magnetic field could be observed, showing two maxima (minima) when the magnetic field is parallel (perpendicular) to the bridge length. This behaviour was attributed to AMR effects inside the $180^{\circ}$ Néel type domain walls, i.e., where the magnetization rotates in the film plane. We demonstrated that the combined effects of CMR and AMR inside domain walls when a multidomain configuration exists can increase the LFMR and sensitivity in a narrow magnetic field range. Finally, we measured the low frequency noise in order to evaluate the magnetic noise spectral density level $b_{n}$. It was about $29 \mathrm{nT} \mathrm{Hz}-1 / 2$ at $1 \mathrm{~Hz}$, which is equivalent to Hall and GMR sensor performance. Further sensor design and electronic readout are possible in order to increase LSMO-based magnetic sensor performance at room temperature.

\section{ACKNOWLEDGMENTS}

The authors wish to thank C. Simon at CRISMAT (UMR6508) for SQUID magnetization measurements and CRISMAT for the LSMO target fabrication.

${ }^{1}$ M. Bowen, M. Bibes, A. Barthélémy, J.-P. Contour, A. Anane, Y. Lemaître, and A. Fert, Appl. Phys. Lett. 82(2), 233 (2003).

${ }^{2}$ A.-M. Haghiri-Gosnet and J.-P. Renard, J. Phys. D: Appl. Phys. 36, R127-R150 (2003).

${ }^{3}$ Y. Tokura and Y. Tomioka, J. Magn. Magn. Mater. 200, 1-23 (1999).

${ }^{4}$ Y. Tokura, Rep. Prog. Phys. 69, 797-851 (2006).

${ }^{5}$ J. Coey, M. Viret, and S. von Molnar, Adv. Phys. 48, 167 (1999).

${ }^{6}$ M. B. Salamon and M. Jaime, Rev. Mod. Phys. 73, 583 (2001).

${ }^{7}$ A. P. Ramirez, J. Phys.: Condens. Matter 9, 8171 (1997).

${ }^{8}$ M. Ziese, Rep. Prog. Phys. 65, 143 (2002).

${ }^{9}$ P. K. Siwach, H. K. Singh, and O. N. Srivastava, J. Phys.: Condens. Matter 20, 273201 (2008).

${ }^{10}$ K.-K. Choi, T. Taniyama, and Y. Yamazaki, J. Appl. Phys. 90, 6145-6150 (2001).

${ }^{11}$ X. W. Li, A. Gupta, G. Xiao, and G. Q. Gong, Appl. Phys. Lett. 71, 1124 (1997).

${ }^{12}$ H. Y. Hwang, S. W. Cheong, N. P. Ong, and B. Batlogg, Phys. Rev. Lett. 77, 2041-2044 (1996)

${ }^{13}$ T. Venkatesan, M. Rajaswari, Z. Dong, S. B. Ogale, and R. Ramesh, Philos. Trans. R. Soc. London, Ser. A 356, 1661 (1998).

${ }^{14}$ N. D. Mathur, G. Burnell, S. P. Issac, T. J. Jackson, B. S. Teo, J. L. MacManus-Driscoll, L. F. Cohen, J. E. Evetts, and M. G. Blamire, Nature (London) 387, 266-268 (1997).

${ }^{15}$ R. Gunnarsson, Z. G. Ivanov, C. Dubourdieu, and H. Roussel, Phys. Rev. B 69, 054413 (2004).

${ }^{16}$ G. Alejandro, L. B. Steren, H. Pastoriza, D. Vega, M. Granada, J. C. Rojas Sanchez, M. Sirena, and B. Alascio, J. Phys.: Condens. Matter 22, 346007 (2010).

${ }^{17}$ A. A. Bosak, C. Dubourdieu, P. Chaudouët, and J.-P. Sénateur, J. Appl. Phys. 94(8), 5021-5025 (2003).

${ }^{18}$ M. Bibes, B. Martinez, and J. Fontcuberta, V. Trtik, F. Benitez, F. Sànchez, and M. Varela, Appl. Phys. Lett. 75, 2120-2122 (1999).

${ }^{19}$ J. Wolfman, A. M. Haghiri-Gosnet, B. Raveau, C. Vieu, E. Cambrill, A. Cornette, and H. Launois, J. Appl. Phys. 89(11), 6955 (2001).

${ }^{20}$ V. Arnal, A. V. Khvalkovskii, M. Bibes, B. Mercy, P. Lecoeur, and A.-M. Haghiri-Gosnet, Phys. Rev. B 75, 220409 (2007).

${ }^{21}$ A. Ruotolo, A. Oropallo, F. Miletto Granozio, G. P. Pepe, P. Perna, U. Scotti di Uccio, and D. Pullini, Appl. Phys. Lett. 91, 132502 (2007).

${ }^{22}$ I. Pallechi, E. Bellingeri, G. Canu, A. Caviglia, A. S. Siri, and D. Marré, J. Appl. Phys. 99, 114508 (2006).

${ }^{23}$ Y. Lu, X. W. Li, G. Q. Gong, G. Xiao, A. Gupta, P. Lecoeur, J. Z. Sun, Y. Y. Wang, and V. P. Dravid, Phys. Rev. B 54, R8357-R8360 (1996).

${ }^{24}$ M. Viret, M. Drouet, J. Nassar, J. P. Contour, C. Fermon, and A. Fert, Europhys. Lett. 39, 545-549 (1997).

${ }^{25}$ S. S. P. Parkin, C. Kaiser, A. Panchula, P. M. Rice, B. Huches, M. Samant, and S.-H. Yang, Nature Mater. 3, 862-867 (2004). 
${ }^{26}$ A. Gupta, G. Q. Gong, G. Xiao, P. R. Duncombe, P. Lecoeur, P. Trouilloud, Y. Y. Wang, V. P. Dravid, and J. Z. Sun, Phys. Rev. B 54, R15629-R15632 (1996).

${ }^{27}$ P. Lecoeur, P. L. Trouilloud, G. Xiao, A. Gupta, G. Q. Gong, and X. W. Li, J. Appl. Phys. 82, 3934-3939 (1997).

${ }^{28}$ M. Saïb, M. Belmeguenai, L. Méchin, D. Bloyet, and S. Flament, J. Appl. Phys. 103, 113905 (2008)

${ }^{29}$ P. Perna, L. Méchin, M. Saïb, J. Camarero, and S. Flament, New J. Phys. 12, 103033 (2010).

${ }^{30}$ E. J. Kim, J. L. R. Watts, B. Harteneck, A. Scholl, A. Young, A. Doran, and Y. Suzuki, J. Appl. Phys. 109, 07D712 (2011).

${ }^{31}$ J. Rhensius, C. A. F. Vaz, A. Bisig, S. Schweitzer, J. Heidler, H. S. Körner, A. Locatelli, M. A. Ninõ, M. Weigand, L. Méchin, F. Gaucher, E. Goering, L. J. Heyderman, and M. Kläui, Appl. Phys. Lett. 99, 062508 (2011).

${ }^{32}$ P. Perna, C. Rodrigo, E. Jiménez, F. J. Teran, N. Mikuszeit, L. Méchin, J. Camarero, and R. Miranda, J. Appl. Phys. 110, 013919 (2011).

${ }^{33}$ M. Koubaa, A. M. Haghiri-Gosnet, J. P. Renard, M. Veis, V. Kolinsky, S. Visnovsky, Ph. Lecoeur, W. Prellier, and B. Mercey, J. Magn. Magn. Mater. 272, 1812 (2004).

${ }^{34}$ L. Méchin, J.-M. Routoure, S. Mercone, F. Yang, S. Flament, and R. A. Chakalov, J. Appl. Phys. 103, 083709 (2008).

${ }^{35}$ J.-M. Routoure, D. Fadil, S. Flament, and L. Méchin, AIP Conf. Proc. 922(1), 419-424 (2007).

${ }^{36}$ C. Shearwood, S. J. Blundell, M. J. Baird, J. A. C. Bland, M. Gester, H. Ahmed, and H. P. Hughes, J. Appl. Phys. 75, 5249 (1994).

${ }^{37}$ G. Bayreuther, P. Bruno, G. Lugert, and C. Turtur, Phys. Rev. B 40, 7399 (1989).

${ }^{38}$ T. Thomason, K. O'Grady, and G. Bayreuther, J. Phys. D: Appl. Phys. 30, 1577-1587 (1997).
${ }^{39}$ M. Viret, D. Vignoles, D. Cole, J. M. D. Coey, W. Allen, D. S. Daniel, and J. F. Gregg, Phys. Rev. B 55, 8464 (1996).

${ }^{40}$ T. R. Mcguire and R. I. Potter, IEEE Trans. Magn. 11(4), 1018-1038 (1975).

${ }^{41}$ X. Hong, J.-H. Yau, J. D. Hoffman, C. H. Ahn, Y. Bason, and L. Klein, Phys. Rev. B 74, 174406 (2006).

${ }^{42}$ J. O'Donnell, M. Onellion, M. S. Rzchowski, J. N. Eckstein, and I. Bozovic, Phys. Rev. B 55(9), 5873-5879 (1997).

${ }^{43}$ M. Ziese and S. P. Sena, J. Phys.: Condens. Matter 10, 2727-2737 (1998).

${ }^{44}$ M. Egilmez, K. H. Chow, and J. A. Jung, Mod. Phys. Lett. B 25(10), 697 (2011).

${ }^{45}$ J. D. Fuhr, M. Granada, L. B. Steren, and B. Alascio, J. Phys.: Condens. Matter 22, 146001 (2010).

${ }^{46}$ A. Hubert and R. Schäfer, Magnetic Domains (Springer, Berlin/Heidelberg/New York, 1998).

${ }^{47}$ R. Akiyama, H. Tanaka, T. Matsumoto, and T. Kawai, Appl. Phys. Lett. 79(26), 4378 (2001).

${ }^{48}$ M. Ziese, Phys. Status Solidi B 242, R116 (2005).

${ }^{49}$ F. N. Hooge, Phys. Lett. 29, 139-140 (1969).

${ }^{50}$ R. S. Popovic, P. M. Drljaca, and C. Schott, in Proceedings of the $23 \mathrm{rd}$ International Conference on Microelectronics (MIEL, 2002), Vol. 1, p. 55.

${ }^{51}$ See http://www.nve.com/Downloads/apps.pdf for more information about NVE GMR sensor.

${ }^{52}$ X. Zhuang, C. Cordier, S. Saez, M. Lam Chok Sing, C. Dolabdjian, J. Gao, J. F. Li, and D. Viehland, J. Appl. Phys. 109, 124512 (2011)

${ }^{53}$ E. Weiss and E. Paperno, J. Appl. Phys. 109, 07E529 (2011).

${ }^{54}$ M. Malátek and L. Kraus, Sens. Actuators, A 164(1-2), 41 (2010).

${ }^{55}$ H. Duan, H. W. Tseng, Y. Li, and R. B. van Dover, J. Appl. Phys. 109, 113917 (2011). 